II.

\title{
Eine angebliche Urkunde Papst Gelasius' II. für die Regularkanoniker.
}

\author{
Von \\ Herrn Prof. Dr. Wilhelm Levison \\ in Bonn.
}

Die im folgenden mitgeteilte Lrkunde findet sich auf einem Pergamentblatt, das Pfarrer D. Carl Erbes in Castellaun vom Einband eines Cölner Druckes, des sogenannten Ambrosiasters, aus dem Jahre 1530 losgelöst hat, den er 1882 von dem Antiquariat Windprecht in Augsburg erworben hatte. $\left.{ }^{1}\right)$ Der Band gehörte vordem den Karmelitern zu Dinkelsbühl in Mittelfranken laut der Eintragung auf dem Titelblatt: 'Sum ff. ( $=$ fratrum) Carmel. Dinckelsp.'; unten hat dort noch ein späterer Besitzer: 'Jos. Müller Pf(arrer)' seinen Namen verzeichnet. Das Pergamentstück hat Herr Erbes Herrn Professor D. Dr. Hans Achelis in Bonn geschenkt, der es der dortigen Universitätsbibliothek zu übergeben beabsichtigt und mich zu dieser Veröffentlichung veranlaßt hat.

Das Stück besteht aus zwei von je einer Hand des 12. Jahrhunderts in Bücherschrift beschriebenen Blättern, die zusammengenäht worden sind, um dem Einband jenes Druckes als äußere Hülle zu dienen, und die durch Beschneiden und an der Außenseite durch Abreiben der Schrift stark gelitten haben. Das größerc Bruchstück, das den hinteren Deckel und den Rücken ${ }^{2}$ ) bekleidete und mit

1) 'Divi Ambrosii episcopi Mrdiolanensis commentarii in omnes Divi Pauli epistolas, ex restitutione D. Frasmi diligenter recogniti. Coloniae Apud Ioannem Gymnicum An. M.D.XXX. mense Ianuario.' Die Angaben über die Herkunft des Blittes verdanke ich einer freundlichen Mitteilung von Herm Pfarrer Erbes.

2) Hier ist noch ein Schildehen mit den Bibliothekszeichen (ron Dinkelsbühl ?) B aufgeklebt. 
einem Rande noch auf den Vorderdeckel hinübergriff, um- faßt ein Doppelblatt, dessen eine Hälfte allerdings zum 1 großen Teil abgeschnitten ist, und enthält Bruchstücke? aus dem 4. Buch eines verkürzten Textes von Amalars s Werk De ecclesiasticis officiis. ${ }^{1}$ ) Daran ist ein kleineres ; Blatt (jetzt $16 \times 8,5 \mathrm{~cm}$ ) angenäht, das den größeren Teil 1 des Vorderdeckels schützte und die Abschrift einer Papst- urkunde enthält. Durch Abschneiden ist vorn die rechte, hinten die linke Seite unvollständig geworden, ebenso unten । die letzte Zeile der Vorderseite weggefallen (der untere: Teil der Rückseite ist unbeschrieben); dennoch läßt sich. fast überall mit Sicherheit der volle Wortlaut herstellen, den ich zunächst mitteile. Bei den Ergänzungen ist zu । beachten, daß der Schreiber die üblichsten Abkürzungenı verwendet und, wie die Rückseite zeigt, das Zeilenende: nicht gleichmäßig ausgenutzt hat, so daß die Zahl der ver-lorenen Buchstaben am rechten Rande der Vorderseite ein । wenig schwanken kann. Der Anfangsbuchstabe $G$ ist durch เ besondere Größe und rote Farbe hervorgehoben.

Papst Gelasius (II.) schreibt allen Kanonikern, wie : dankbar er Gott sei für die Erneuerung des kanonischen . Lebens nach der Weise der Urkirche, das derselben Wurzel wie das Mönchtum entsprossen sei, bestätigt den Lebensplan der Kanoniker, erteilt allen, die sich in ihren Stiftern

1) Auf der stark abgeriebenen Vorderseite der vollständigeren Blatthälfte sind Teile von IV 10 (Migne 105 Sp. 1191) erkennbar, auf der Rückseite findet sich der Rest von IV 10 ron '[soc]ietatem. Deinde sequitur lectio pro admonitione' an (eld. Z. 1 von unten), dann IV 11 ('De cottidianis nocturnis') und von 12 ('De cottidianis matutinis') der Anfang (bis in Sp. 1193 hinein). Der andere Teil des Doppelblattes, von dem kaum die linke Hälfte erhalten ist, beginnt in IV 29 von [septi]formis [operatio - congruere]. Et iterum: Octava' bis 'a solis occubitu usque [ad solis occubitunl]' (Sp. $1216 \mathrm{Z} .3$ ron unten bis Sp. 1218); es schließt sich auf der Vorderseite noch der Anfang von IV 37 (Sp. 1229) an: 'Ubi sanctus Ieronimus cxponit il[lud ad Galatas: Dies observatis et mens]es et tempora et annos. Tim[eo]', gleichwie sich auf der Rückseite weitere Reste desselben Kapitels (bis Sp. 1230) erkennen lassen. Herr P. Cunibert Mohlberg O. S. B. aus Maria-Laach hat mich beim Ermitteln des Textes auf die rechte Spur gebracht, wofür ibm auch hier bestens gedankt sei. 
darauf verpflichten, Segen und Absolution, verbietet Änderung ihrer Lebensordnung und das Verlassen des Claustrums ohne Erlaubnis von Propst und Kongregation und macht die Zulässigkeit der Aufnahme an anderen Orten von dem Vorliegen eines Erlaubnisschreibens abhängig.

1118-1119.

Gelasius servus servorum Dei omnibus in can[onico ordine secundum]: 2 Deum virentibus salutem et gratiam in Christo Iesu [atque coepti sacri ordinis] ${ }^{3}$ nitidam perseveranter ${ }^{1}$ ) observare puritatem. Sancto [Spiritui gratias agimus], i cuius instinctu circa canonicum ordinem vos, fr[atres], ${ }^{5}$ fervorem et studium habere cognoscimus. O[mnipotenti] 6 Domino, cuius melior est misericordia super vitas $^{2}$ ), gratias agim[us, quia vos estis, qui] $\left.\right|^{7}$ sanctorum patrum vitam probabilem renovatis et apo[stolice insti]$\left.\right|^{8}$ tuta doctrinę, primordiis ęcclesię sanctę inolita, sed cre$s\left[\right.$ cente ęcclesia iam] ${ }^{9}$ pene deleta, instinctu sancti Spiritus suscitatis. Duę [enim ab ęcclesię sanctę $]{ }^{10}$ primordiis vitę eius sunt filiis institutę: una, qua i[nfirmorum debi]11litas retinetur, altera, qua forciorum virtus beata [perficitur; una] ${ }^{12}$ remanens in Segor parvula, altera ad montis [altiora conscendens] $\left.{ }^{3}\right) ;\left.\right|^{13}$ una lacrimis et elemosinis cottidiana peccata [redimens, altera] ${ }^{14}$ cottidiana instantia merita etterna conquirens. [Alteram tenentes] 15 inferiorem bonis terrenis utuntur, alteram sequen[tes superiorem] $\left.\right|^{16}$ bona terrena despiciunt ac derelinquunt. [Hęc autem, quę] ${ }^{17}$ a terrenis divino fervore divertitur, in duas [unius pene eiusdemque] ${ }^{18}$ propositi dividitur portiones, canonicorum atque monac[horum. Harum ${ }^{19} \mathrm{se}-$ cunda] per divinam misericordiam frequentata satis iam se[culo universo elu- $\mid{ }^{20}$ cet; pri]ma vero, decalescente fervore ${ }^{4}$ ) fidelium, iam pe[ne omnino de- $]\left.\right|^{21}$ fluxit. Hanc martyr et pontifex Urbanus insti[tuit $\left.{ }^{5}\right)$, hanc $\left.\mathrm{Au}-\right] \mid{ }^{22} \mathrm{gu}-$

1) 'perseverantur' Hs. $\quad$ 2) Psalm 62, 4.

3) Vgl. Genesis 19, V. 17-22 und 30 .

4) Eine neuere Hand hat an dem abgeriebenen Worte herum. gebessert, das jetzt wie 'fertoris' aussieht.

5) Vgl. Decretales Pseudo-Isidorianae ed. Hinschius S. $143 \mathrm{f}$. 
stinus suis regulis ordinavit, hanc suis epistol[is Hieronimus] ${ }^{23}$ informavit. Non minoris itaque estimandum est mer[iti vitam hanc] ${ }^{24}$ ęcclesię primitivam, aspirante ac prosequente Domini spiritu, su[scitare quam] $\left.\right|^{25}$ florentem monachorum religionem eiusdem spiritus perseve[rantia custo-] ${ }^{26}$ dire. Vestrum ergo propositum nostri auctoritate offic[ii confirmamus et ${ }^{27}$ fir]mos vos [in eo] ${ }^{1}$ ) persistere adh[ortamur et tamquam Deo exhortante per nos] ${ }^{28}$ obsecramus. Quam ob rem omnibus in cenobiis ${ }^{2}$ ) [vestris vitam canonicam secundum huius tenorem ordinis ${ }^{3}$ ) profitentibus et in ea, adiuvante Domino, ${ }^{1}$ permanentibus nos licet i]ndigni apostolorum vicarii eorum ac nostram benedictio- $\mid{ }^{2}$ [nem peccatorumque abs]olutionem, potestate illis a Domino indulta, concedimus, ${ }^{3}$ [constituentes, ne cu ]iquam liceat omnino hunc vestri statum [ordinis] ${ }^{4}$ ) commutare, $\left.\right|^{4}$ [cuius tantus in tot ter]rarum partibus fructus exuberat, ut plures ${ }^{5}$ [ęcclesię vestri saporis] dulcedine condiantur. Statuimus etiam, ne $\left.\right|^{6}$ [professionis canon]ice quispiam, postquam Dei vice super caput sibi | [hominem imposue $]$ rit $^{5}$ ), alicuius levitatis instinctu vel distric- ${ }^{8}$ [tioris religionis] obtentu ex eodem claustro audeat sine praepositi $\left.{ }^{6}\right) \mid{ }^{9}$ [totiusque congregati]onis permissione discedere; discedentem vero ut $\left.\right|^{\mathbf{1 0}}$ [nullus abbatum] vel episcoporum,

1) 'in eo' fehlt in der Hs.

2) Die nächsten Buchstaben sind nicht mehr lesbar, und die letzto Zeile der Seite ist abgeschnitten; mit ' ....ndigni' beginnt die Rückseite, deren linker Rand ebenfalls durch Wegschneiden unvollständig geworden ist.

3) So die Privilegien Crbans II. für Rottenbuch und St. Rufus und die Legatenurkunde von 1124; 'secundum beati Augustini regulam profitentibus' die Lrkunde Urbans für Maguelonne und die Calixts II. für Mende (s. unten über diese Urkunden).

4) 'ordinis' fehlt in der Hs.

5) Vgl. die Regel Benedikts c. 2 über den Abt: 'Christi enim agere vices in monasterio ereditur' (ebenso c. 63) und c. 7: 'Et ut ostendat sub priore debere nos esse, subsequitur dicens: In posuisti homines super capita nostra' (Psalm 65, 12).

-) So auch die Privilegien für Rottenbuch, Maguelonne und Mende, während die Urkunden Urbans II. für St. Rufus und St. Quentin bei Beauvais und die Legatenurkunde für Norbert 'abbatis' statt 'praepositi' haben. 
Eine angebl. Urkunde Papst Gelasius' II. f. d. Regularkanoniker. 31

nullus monachorum sine communium $/{ }^{11}$ [litterarum cautio]ne suscipiat, auctoritate Dei et nostra interdicimus. 12 [Vos ergo, filii in Christo] carissimi, dilectioni nostrę studiis semper prestan- ${ }^{13}$ [tioribus respond] entes, strennue quę Deo promisistis implere sata- ${ }^{11}$ [gite. Luceat lux] vestra coram hominibus, ut videant vestra bona ${ }^{15}$ [opera et glorific]ent Patrem vestrum, qui in celis est. ${ }^{1}$ ) Cuius $\mathrm{Pa}-$ tris ${ }^{16}[$ hęc, ut firma per]maneant, et Filii et Spiritus sancti virtute sancimus.

Der Rest der Seite, jetzt noch etwa elf Zeilen, ist freigelassen.

Wenn sich der trümmerhafte Text der Urkunde so gut wie sicher ergänzen läßt, so deshalb, weil mehrere andere Privilegien größere oder kleinere Teile des gleichen Wortlautes aufweisen. Ich kann folgende anführen, ohne damit Anspruch auf Vollständigkeit erheben zu wollen ${ }^{2}$ ):

1. Urban II. für das Chorherrenstift Rottenbuch (Raitenbuch) im Bistum Freising 28. Januar 1092 (Jaffé $I^{2}$ Nr. 5459; Brackmann, Germania pontificia I 375 Nr. 2). ${ }^{3}$ ) Die Urkunde wiederholt in der Hauptsache ein älteres Privileg von 1090 (Jaffé Nr. 5428; Brackmann a. a. O. Nr. 1) ${ }^{4}$ ), in dessen Wortlaut das unserem Text entsprechende Stück mitten hineingeschoben ist. Lediglich dem Privileg von 1092 sind die Sätze entnommen, die Gerhoh von Reichersberg in seinem Kommentar zum 64. Psalm mitgeteilt (MG. Libelli de lite imp. et pontif. III 474) und mit den Worten eingeleitet hat: 'Multo rectius obtulit

1) Natth. 5, 16.

2) Von päpstlichen Privilegien für Regularkanoniker mit sachlich in dem einen oder andern Punkte übcreinstimmendem, aber in der Fassung trotz kleiner Anklänge atweichendrm Formular sehe ich absichtlich ab, so von den Urkunden Trbans II. für die Kanoniker von Cahors, Avignon und St. Rufus von 1095 (Jaffé $I^{2}$ Nr. 5573, 5578, 5579; Migne 151 Sp. 423, 426, 427, die letzte Urkunde auch bei Chevalier, Codex diplomaticus S. Rufi [ $\because$ S. 32 Anm. 3] S. 9 Nr. 6).

$\left.{ }^{3}\right)$ Monumenta Boica VIII 8 ff.; Migne 151, 337 ff. Vgl. A. Brackmann, Studien und Vorarbeiten zur Crormania pontificia I, 1912, S. $14 \mathrm{ff}$.

4) J. v. Pflugk-Harttung, Aeta pontificum Romanorum inedita II 146 Nr. 180. 
rectiusque divisit Urbanus papa II. de ordine canonico) scribens regularibus clericis in hunc modum.' Hat manı auch bisher die Zugehörigkeit des nun folgenden *Stückes; zu dem Privileg für Rottenbuch nicht erkannt und darinı ein selbständiges Schreiben Urbans geseben (Jaf fé Nr.5761), so finden sich doch alle Sätze in jener Urkunde von 1092: wieder, der Anfang ('Oportet nos, qui - videant equita-. tem') in der Arenga des auf das Privileg von 1090 zurück-gehenden Teils, der Rest ('Omnipotenti ergo Domino perseverantia custodire') in den hier in Betracht kommen. den Zusätzen von 1092, und eine ganze Reihe von Lesarten bestätigt die Abhängigkeit Gerhohs von dem zweiten Privileg für Rottenbuch.1) Zur Erklärung genügt es, daran zu erinnern, daß der spätere Propst von Reichersberg eben in jener Gründung Herzog Welfs in den zwanziger Jahren zweimal eine Zuflucht gefunden hatte. ${ }^{2}$ ) Sein Text hat also neben dem Privileg von 1092 keine selbständige Bedeutung.

2. Urban II. für den Abt Arbert des Chorherrenstiftes St. Rufus bei Avignon 1088/99 (Jaffé $\mathrm{I}^{2} \mathrm{Nr} .5763$ ). ${ }^{3}$ ) Ein Bruchstuick daraus ('Statuimus, ne professionis - cautione suscipiat') ist durch Vermittelung Ivos von Chartres ${ }^{4}$ ) in Gratians Decretum aufgenommen worden (C. XIX qu. 3 c. 3).

1) Den Zusammenhang des von Gerhoh gebotenen Textes (G) mit . dem bisher nicht ganz fehlerfrei gedruckten Privileg für Rottenbuch (R) zeigen folgende Worte, bei denen ich die entsprechenden Losarten der übrigen vergleichbaren Urkunden voranstelle: doctrinae] 'disciplinae' GR; inolita] 'exorta' GR; bonis terrenis] 'terrenis bonis' GR; informavit] 'hanc Gregorius Augustino Anglorum archiepiscopo instituendam praecepit' fügen GR hinzu.

2) Vgl. Sackur, MG. Libelli UI $131 \mathrm{f}$.

3) Veröffentlicht von Ulysse Che valier, Codex diplomaticus ordinis Sancti Rufi Valentiae (Collection de cartulaires dauphinois IX), 1. Lieferung, Valence 1891, S. 8-9 Nr. 5. Ich weiß nicht, weshalb Chevalier die, soweit ich sehe, nur durch die Zeit Urbans II. (1088-99) datierbare Urkunde in die Jahre 1092-99 gesetzt hat; doch ist der Ansatz wohl richtig, wie man vielleicht aus einer Abweichung gegenüber dem Privileg für Rottenbuch schließen darf, vgl. unten S. $37 f$.

4) Decretum VI 411 (Migne 161, 533). P. Ewald, Neues Archiv $\nabla 372$ Anm. 6 hat irrtümlich die Quelle in dem anderen Privileg Urbans für St. Rufus von 1095 (Jaffé I² Nr. 5579; s. oben S. 31 Anm. 2) gesehen. 
Eine angebl. Orkunde Papst Gelasius' II. f. d. Regularkanoniker. 33

3. Urban II. für das Chorherrenstift St. Paul bei Narboonne 19. März 1093 (Jaffé Nr. 5482). ${ }^{1}$ )

4. Urban II. für das Chorherrerstift St. Quentin bei Beauvais 11. November 1093 (Jaffé Nr. 5496). ${ }^{2}$ )

5. Urban II. für die Kanoniker von Maguelonne 114. März 1095 (Jaffé Nr. 5550). ${ }^{3}$ )

6. Calixt II. für die Kanoniker von Mende 1123 (Jaffé NNr. 7025). ${ }^{4}$ )

7. Urkunde zweier Kardinallegaten, des Presbyters Petrus Leonis und des Diakons Gregor von S. Angelo, für Norbert (den Stifter des Prämonstratenserordens) und die ilhm untergebenen Kanoniker, Noyon 28. Juni 1124 (Le Paige, Bibliotheca Praemonstratensis ordinis I, 1633, S. 390f.; Hugo, Ordinis Praemonstratensis Annales I, 11734, Probationes S. VIIIf.).5)

1) De vic et Vaissete, Histoire générale de Languedoc ${ }^{2} \mathrm{~V}$ (1875) 7:25ff.; Migne 151, $360 \mathrm{f}$.

2) Analecta juris pontificii, $X^{e}$ série (vol. V 2), 1869, Sp. $531 \mathrm{ff}$. Das Stift St. Quentin hatte früher der um die Emeuerung des kanonisichen Lebens so verdiente Ivo geleitet, ehe er Bischof von Chartres wurde.

3) Migne 151, $408 \mathrm{ff}$.

4) Ul. Ro bert, Bullaire du pape Calixte II, 1891, Bd. II 126 Nr. 355.

5) Die Drucke geben das Inkanationsjahr 1125, und so setzt z. B. moch Hauck, Kirchengeschichte Deutschlands IV 3.4 (1913) 374 die Legatenurkunde an. Doch weist das 6. Pontifikatsjahr Calixts II. auf 1124, wie denn der Papst bereits am 14. Dezember dieses Jahres ges.torben ist (vgl. z. B. Neues Archiv XXXV 413 Anm. 6), und so haben bereits Jos. Scholz. Vita St. Norberti, Pars prior (Breslauer Dissertiation), 1859, S. 33 Anm. 80 und God. Madelaine, Histoire de saint Norbert, Lille 1886, S. 266 Anm. 1 mit Recht MCXXV in MCXXIV verbessert, zumal über die Tätigkeit derselben beiden Legaten in Frankreich Ende 1123 und im Jahre 1124 auch andere Nachrichten vorliegen; rgl. R. Zoepffel, Die Papstwahlen, 1871, S. 298f. und Hefele-Leclerc $q$, Histoire des canciles V 1, 1912, S. 648f. Zu derselben Legation ron 1123/24 gehört offenbar die von W. Wiederhold, Papsturkunden in Frankreich VI (Nachrichten der Göttingischen Gesellschaft der Wissenschaften, Phil.-hist. Klasse 1911, Beiheft) S. 27 Nr. 5 veröffentlichte und um 1120 angesetzte Urkunde der beiden Legaten für. Peter von Cluny und die Mönche von Montierneuf. An die Bestätigung von Norberts Orden bei Gelegenheit der Gallischen Legation hat Pierleoni als Anaklet II. 1130 den damaligen Erzbischof von Magdeburg erinnert; s. sein Schreiben bei Giesebrecht, Geschichte der deutschen Kaiserzeit IV ${ }^{2}$, 1877, S. 505 (Jaffé $I^{2}$ Nr. 8409).

Zeitschrift für Rechtsgeschichte. XXXIX. Kan Abt. VIU. 
Diese sieben Privilegien für Augustinerchorherren entthalten in folgender Weise Abschnitte, die der vorstehendern Urkunde vom zweiten Satze des Kontextes an entsprechenı:

Omnipotenti Domino - - Spiritus suscitatis.

Duae enim ab - - altiora conscendens; una lacrimis - - aeterna conquirens. Alteram tenentes - - perseverantia custodire.

Vestrum ergo propositum - - per nos obsecramus.

Quam ob rem omnibus - - indulta concedimus, constituentes, ne cuiquam liceat - - statum ordinis commutare,

cuius tantus in tot - - dulcedine condiantur. $\mathbf{1})$

Statuimus etiam - - litterarum cautione suscipiat,

auctoritate Dei et nostra interdicimus. ${ }^{2}$ ) Vos ergo, flii - virtute sancimus.

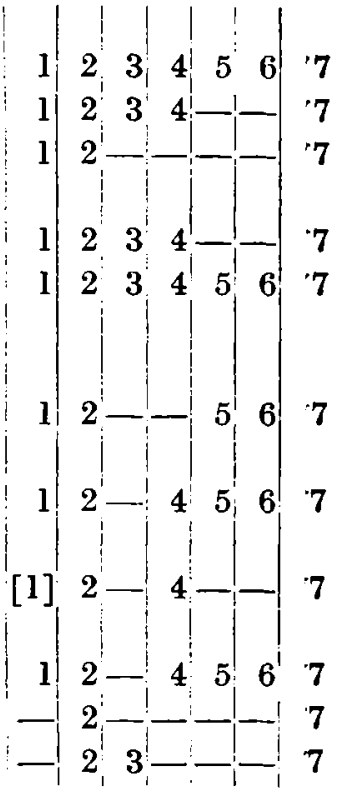

Nur die Anfangsworte 'Omnipotenti Domino, cuius melior est misericordia super vitas', kehren in einer Urkunde von Innocenz II. für Pontigny von 1139 wieder ( $J$ affé $\mathrm{I}^{2}$ Nr. 7959) ${ }^{3}$ ), während ein Mandat desselben Papstes an die Kanoniker von Berchtesgaden aus den Jahren 1138/43 und ein zugehöriges Schreiben an den Erzbischof Konrad von Salzburg (Jaffé Nr. 8294/95; Brackmann I 61 Nr. 6 und 7 , vgl. S. 23 Nr. 63) ${ }^{4}$ ) die Aufrechterhaltung der Augustiner-

1) Über die abweichende Fassung von 1 vgl. unten S. 38.

2) Die Urkunde Crbans für St. Rufus (2) und die Legatenurkunde (7) haben nur das Wort 'interdicimus'; 'auctoritate Dci et nostra' fehlt darin.

3) Migne 179, 412.

4) Die Urkunde für Berchtesgaden bei Pflugk-Harttung a. a. O. II 319 Nr. 355; das Schreiben an Konrad von Salzburg haben Gerhoh von Reichersberg im Kommentar zum 64. Psalm (MG. Libelli III 456f.) und sein Bruder und Nachfolger Arno im Scutum canonicorum (Migne $194,1516 \mathrm{f}$.) wiedergegeben. 
regel und das Verbot, sie aufzugeben, mit Worten aussprechen und begründen, die in größerem Umfang mit den hier besprochenen Urkunden übercinstimmen. Und das Verbot, das Claustrum nach der Profe $B$ olne Erlaubnis der Kongregation und ihres Vorstehers zu verlassen, und die Forderung eines Erlaubnisschreibens vor der Aufnahme in einen anderen Konvent ist in nahezu dem gleichen, wenn auch verkürzten Wortlaut in zahlreiche Privilegien für Augustiner, Prämonstratenser und a uch für andere Orden übergegangen und ein fester Bestandteil des Privilegienformulars geworden. ${ }^{1}$ ) Einen Anklang an die Ausführungen über die Geschichte der 'vita canonica' darf man vielleicht auch darin erkennen, wenn Paschalis II. 1109 den Kanonikern von S. Frediano in Lucca, als er die Exkonmunikation eines gegen das Austrittsverbot sündigenden Presbyters bebestätigte ${ }^{2}$ ), von der 'vita regularis' sagte: 'Hanc beatus Augustinus tam gratanter amplexus est, ut eam suis regulis informaret'; desgleichen, wenn bei Gelegenheit der Annahme der Augustinerregel durch Norbert und seine Gefährten in Prémontré, die Weihnachten 1121 erfolgte, sein Biograph berichtet ${ }^{3}$ ): 'Sed ille _ _ regulam, quam beatus Augustinus suis instituit, afferri praecepit. Apostolica etenim vita - - iam optabat vivere, quam utique ab eodem beato viro post apostolos audierat ordinatam et renovatam fuisse.'

So weist unsere Urkunde einen Wortlaut auf, der einst offenbar weit verbreitet gewesen ist und von dem sicherlich sich noch weitere Spuren finden lassen, als es hier geschehen ist. ${ }^{4}$ ) Er führt zurück in die Frühzeit des erneuerten

1) M. Ta ngl, Die päpstlichen Kanzleiordnungen, 1894, S. 230 (I 8), :33 (111 8), 306 (CIV 8); vgl. Grorg Sichreiber, Kurie und Kloster in 12. Jahrhundert II (Stutz, Kirehenrechtliche Abhandlungen 67/68), 1910, S. $341 \mathrm{ff} ., 369,372,374 \mathrm{f}$. und von drn vielen Belegen z. B. Urban II. 2095 für Standalmont (Jaffé 5567; Migne 151, 419), Calixt II. 1121 für Berchtesgaden (Jaffé 6903; Brackmann I 60 Nr. 2; Roberta. a. O. : $338 \mathrm{Nr}$. 229), Honorius II. 1126 für Jrémontré (Jaffé 7244; Migne $66,1249)$.

2) Jaffé 6492; Kehr, Italia pontificia III 415 Nr. 8; Migne .63, 394 .

3) Vita Norberti c. 12 (SS. XII 683).

4) Vgl. auch unten S. 43 Anm. 1. 
kanonischen Lebens der Geistlichkeit, als nach des spätesren Gregors VII. Eintreten zu dessen Gunsten auf derr Lateransynode von 10591) und nachdem er als Papst für Regularkanoniker eine neue Regel verfaßt hatte ${ }^{2}$ ), die zu keinem bedeutenderen Einfluß gelangt zu sein scheint, in derr sogenannten Regel Augustins eine Norm des gemeinsamem Lebens für den Klerus aufkam, die größere Verbreitung fand und an zahlreichen Orten das Vorbild der Mönchee auf die Kanoniker übertragen half ${ }^{3}$ ), nicht zum wenigstem in Baiern, aus dessen Nachbargebieten unsere Abschriftt herzustammen scheint. ${ }^{4}$ ) So wird denn hier die Art solcherr Kanoniker als dem Mönchtum gleichberechtigte Äußerung desselben Strebens hingestellt, wird die Lebensordnung der Regularkanoniker bestätigt, eine Änderung derselben verboten und werden jene Bestimmungen erlassen über die Unzulässigkeit des Austritts und die Vorbedingung der Aufnahme durch Bischöfe, Äbte und Mönche eines andern Konvents. Der Wortlaut in der hier vorliegenden Fassung geht, wie die genannten Urkunden zeigen, im wesentlichen bis mindestens 1092 zurück, bis in die früheren Jahre Urbans II., dessen Zeit ,,die wichtigen Anfänge eines erster primitiven Formulars für die Regularkanoniker" angehö-

1) S. die Akten von 1059 bei Mabillon, Annales ordinis S. Benedicti IV $748 \mathrm{ff}$. und Werminghoff, Neues Archiv XXVII $669 \mathrm{ff}$; rgl. ebd. S. 644 und Hauck a. a. 0. ${ }^{3.4}$ III 697; IV 356.

2) Herausgegeben von G. Morin, Rerue Bénédictine XVIII (1901) $177 \mathrm{ff}$; Études, textes, découvertes (Anecdota Maredsolana, Seconde série) I, 1913, S. $457 \mathrm{ff}$.

3) Vgl. u. a. Ha u cka. a. O. IV 355 ff.; M. Heim bucher, Die Orden und Kongregationen der katholischen Kirche $\mathrm{II}^{2}$, 1907, S. 1 ff.; E. Speakman, The rule of St. Augustine (Historical essays by members of the Owens College, Manchester, edited by Tout and Tait, London 1902, S. 57-75; Arthur Nebel, Die Anfänge und die kirchliche Rechtsstellung des Augustinerchorherrenstifts St. Peter auf dem Lauterberge, Dissertation von Halle 1916, S. 34 ff. (= Thüringisch-Sächsische Zeitschrift für Geschichte und Kunst VI $136 \mathrm{ff}$.); L. Fischer, Ivo von Chartres, der Erneuerer der Vita canonica in Frankreich (Festgabe Alois Knöpfler gewidmet, Freiburg i. Br. 1917, S. 67-88); Schreiber a. a. O. 1 (Stutz, Kirchenrechtliche Abhandlungen 65/66), 1910, S. $100 \mathrm{ff}$; II $326 \mathrm{ff}$.

4) Vgl. oben S. 27. 
Eine angebl. Urkunde Papst Gelasius' II. f. d. Regularkanoniker. 37

ren. $\left.{ }^{1}\right)$ Der neue Text trägt an der Spitze den Namen des Gelasius, der nur der zweite Papst dieses Namens sein kann, dessen kurzes Pontifikat sich vom Januar 1118 bis zum selben Monat des nächsten Jahres erstreckte; aber in der Hauptsache geht der Text unzweifelhaft auf eine Vorurkunde Urbans zurück, deren Wortlaut er vollständig im Zusammenhang bewahrt hat. Am nächsten steht ihm die Urkunde Urbans für St. Rufus (2), sodann die vielleicht von dieser abhängige Legatenurkunde von 1124 (7), die jedoch entsprechend der untergeordneten Stellung der Legaten einige nur im Munde des Papstes passende Worte geändert und am Ende des Kontexts eine Pönformel angefügt hat. Das Privileg Urbans für Rottenbuch (1) mußte auf die Schlußwünsche ('Vos ergo, filii' usw.) verzichten, da der Text hier einem älteren Privileg eingefügt wurde, das schon ähnliche Wendungen enthielt. Das Privileg für St. Paul bei Narbonne (3) ersetzte den mittleren Teil, das für St. Quentin bei Beauvais (4) die Mitte und den SchluBteil durch andere Bestimmungen. Endlich die Urkunden für Maguelonne (5) und Mende (6) lassen die gesamten Betrachtungen über die Gleichwertigkeit der Regularkanoniker und Mönche beiseite und geben an Stelle der Schlußwünsche nahezu gleichlautende umfangreiche Bestimmungen verschiedener Art, von denen solche über das Verhalten gegenüber Säkularkanonikern, die beim Übergang zum regularen Leben bereits der Gemeinschaft angehört, aber sich der strengeren Richtung nicht angeschlossen haben, auch schon in dem Narbonner Privileg dem ursprünglichen Bestande zugewachsen sind ('Quia vero in vobis - - victurum professus fuerit').

Die den Augustinerchorherren im allgemeinen geltende Fassung, wie sie in der Urkunde des Gelasius auf uns gelommen ist, findet so ein fast ganz entsprechendes Gegenstück nur in der Urkunde Urbans für St. Rufus, deren Wortlaut sicherlich im wesentlichen an die Spitze dieser Ủberlieferungsreihe gehört. Allerdings nur im wesentlichen;

1) So Brackmann in seiner Besprechung des genannten Werkes ton Schreiber, Göttingische gclehrte Anzeigen 1913, S. 278. 
denn an einer Stelle zeigt das Privileg für Rottenbuch eine ältere Stufe der Entwicklung. Was hier noch als Hoffnung begegnet:

'ne cuiquam liceat omnino hunc vestri statum ordinis commutare, de quo in Domino confidimus multum fructum excrescere',

erscheint in der Ưrkunde für St. Rufus wie in denen für Beauvais (1093), Norbert (1124) und in der des Gelasius als verwirklicht ${ }^{1}$ ):

'ne cuiquam omnino liceat hunc vestri statum ordinis commutare, cuius tantus in tot terrarum partibus fructus exuberat, ut plures ecclesiae ${ }^{2}$ ) vestri saporis dulcedine condiantur',

gleichwie eine andere Stelle:

'prima vero, decalescente fervore fidelium, iam pene omnino defluxit',

1124 von den Legaten im Hinblick auf die veränderten Verhältnisse also umgestaltet worden ist:

'Prima vero, licet, decalescente fervore fidelium, aliquando pene defluxerit, nostris tamen temporibus, divina inspirante clementia, vigere plurimum coepit.'

So steht der neue Text gleich dem für St. Rufus in der Mitte zwischen den Privilegien von 1092 und 1124. Der Wortlaut des bisher behandelten größeren Teils stimmt bis ins einzelne so gut wie ganz mit der Urkunde Urbans für St. Rufus überein. Von den wenigen unwesentlichen Abweichungen, die zum Teil vielleicht nur der Ủberlieferung zur Last fallen, ist etwa das Fehlen der Worte auctoritate Dei et nostra' bei Urban (vgl. S. 34 Anm. 2) bemerkenswert; wenn Gelasius 'cenobiis [vestris]', und 'praepositi' sagt, wo man bei Urban 'vestro cenobio' und 'abbatis' liest, so braucht nur darauf hingewiesen $\mathrm{zu}$ werden, daß Urban sich an ein einzelnes Stift wendet, Gelasius an die

1) Die Privilegien für Maguelonne und Mende lassen clie Stelle aus.

2) So die Urkunden für St. Rufus und Beaurais, denen der hier zerstörte Text des Gelasius sicherlich entsprach; das Wort ist in der Legatenurkunde für Norbert ausgelassen, wie dies für die Anfänge Prémontrés begreiflich ist. 
Regularkanoniker überhaupt, und daß die Utberlieferung des jüngeren Textes aus Deutschland stammt, wo die Augustinerstifter meist von Pröpsten geleitet wurden im Gegensatz zu der französischen Abtverfassung. ${ }^{1}$ ) Diese Abweichungen scheinen mir aber die Annahme nicht auszuschließen, daß die Urkunde für das später als Haupt einer Kongregation so einflußreiche ${ }^{2}$ ) St. Rufus mittelbar oder unmittelbar als Vorlage des Gelasiustextes gedient hat. Daß Gelasius II. sich auch sonst um Angelegenheiten der Augustinerchorherren bekümmert hat, zeigen zwei Urkunden, in denen er die Möglichkeit von Abweichungen von der Regel und die Frage des Austritts behandelt. ${ }^{3}$ ) So ist es an sich sehr wohl denkbar, daß der Papst eine Urkunde Urbans II. erneuert oder vielmehr ihre einem bestimmten Chorherrenstift geltenden Sätze auf die Gesamtheit aller Regularkanoniker übertragen hat.

Und doch hindert ein Umstand, das Schriftstück unbedenklich als Urkunde Gelasius' II. hinzunehmen; die Schwierigkeiten liegen in dem einleitenden Teil, von dem ich bisher abgesehen habe. Das Privileg der Legaten von 1124 beginnt den Kontext sogleich mit 'Omnipotenti', ebenso Urban II. den in das Privileg für Rottenbuch 1092 neu eingeschobenen Teil. Die fünf anderen Papsturkunden schicken alle die gleiche kurze Arenga voraus: 'Piae voluntatis affectus prosequente debet studio confoveri, ut ecclesiastica utilitas apostolicae sedis favore vires accipiat et accrescat.' Gelasius läßt dafür dem Dank an Gott einen Dank an den Heiligen Geist voraufgehen, dessen Fassung neben dem nachfolgenden Satz recht unorganisch anmutet.

1) Vgl. Sehreiber a. a. O. II 331.

2) Vgl. Heimbucher a. a. O. S. 23. Schon 1152 wird der 'ordo canonicus secundum Deum et beati Angustini zegulam' in einer Lirkunde Eugens III. 'juxta observantiam patrum Sanoti Ruf' näher bestimmt (Jaffé II ${ }^{2}$ Nr. 9609; Chevalier a. a. O. S. 31 f. Nr. 23). In den Sprengel ron Toul waren die Gewohnheitcn von St. Rufus bereits 1093 oder kurz rorher übertragen worden; rgl. Siheri Primordia Calmosiacensia I (MG. SS. XII 329). Im einzelnen bedaif die Geschichte der Augustinerchorherren noch mancher Aufklärung.

3) Jaffé I ${ }^{2}$ Nr. 6648 (für Springiersbach) und 6649; S. P. Ernst, Histoire de Limbourg VI, 1847, $\leqslant 121 \mathrm{f}$. 
Aber die eigentliche Schwierigkeit ergibt sich aws dem Eingangsprotokoll. Die Urkunde Urbans für St. Rufus bietet hier einen unanstößigen Wortlaut: 'Urbanus episcopus servus servorum Dei dilectis filiis A(rberto) abbati Sancti Rufi eiusque fratribus canonicam vitam professis salutem et apostolicam benedictionem.' Anders die sonst so sehr damit übereinstimmende Gelasiusurkunde. Freilich, daB in dem üblichen Titel 'Gelasius episcopus servus servorum Dei' das Wort 'episcopus' fehlt, kann leicht auf einer Flüchtigkeit des Abschreibers beruhen und wiegt nicht eben schwer. Anders aber liegt es mit der Grußformel. Man erwartet in Privilegien des Gelasius die bereits festgewordene Formel 'in perpetuum', in Briefen 'salutem et apostolicam benedictionem'; dafür liest man hier 'salutem et gratiam in Christo Iesu [atque coepti sacri ordinis] nitidam perseveranter observare puritatem'. Gregor VII. hat als Elekt seinen Briefen den GruB 'salutem in Christo Iesu' oder 'salutem in domino Iesu Christo' vorangestellt ${ }^{1}$ ), um ihn unmittelbar nach der Weihe durch die übliche Formel zu ersetzen; aber auch zwischen diesem Gruß und dem Wunsch des Gelasius besteht ein solcher Unterschied, daß die Annahme einer die Formel Gregors enthaltenden Vorurkunde doch nur einen kleinen Teil der Schwierigkeit beseitigt. Wie nun den folgenden Abschnitten entsprechende Teile sich in anderen Papsturkunden gefunden haben, so gibt es auch ein Gegenstück zu dem Anfang unseres Textes. Letbert, der, nachdem er Kanoniker von St. Peter in Lille gewesen war, zwischen 1095 und 1100 Abt jenes bedeutenden Augustinerstiftes St. Rufus bei Avignon wurde und 1110 oder 1111 gestorben ist ${ }^{2}$, hat außer einem Psalmen-

1) Registrum I 1*. 2. 3. 5--12 (Jaffé, Bibliotheca rerum Germa. nicarum II 10 f.).

2) Über Letbert vgl. Histoire litéraire de la France IX, 1750, S. 570-578; Hauréau, Gallia christiana XVI, 1865, S. $357 \mathrm{f}$. und namentlich Chevalier, Notice littéraire et bibliographique sur Letbert, abbé de Saint-Ruf (Annales de philosophie chrétienne, $5^{\circ}$ série XVI, 1867, S. 214-234), dessen Aufsatz A. We is, Allgemeine Deutsche Bio. graphie XVIII, 1883, S. 458 entgangen ist. Eine zweite Bearbeitung des Aufsatzes von Chevalier im Bulletin de la Société départementale 
Eine angebl. Trrkunde Papst Gelasius' II. f. d. Regularkanoniker. 41

kommentar (*lores psalmorum) namentlich eine kleine Schrift hinterlassen; die er dem Propst Otger der 'Ferranica congregatio'1) und allen Regularkanonikern gewidmet und der Verherrlichung ihres 'canonicus et sacerdotalis ordo' bestimmt hat ${ }^{2}$ ), eine der ersten Schriften zum Preise des Augustinerord'ns. Ihr Eingang nun hat als Quelle für den noch unbrlegten Teil des Gelasiusbriefes gedient:

\section{Let bert.}

Otgerio Ferranicae congregationis venerabili praeposito sanctoque eius conventui et omnibus in canonico ordine secundum Deum viventibus Lambertus ${ }^{3}$ ) humilis ecclesiae Sancti Rufi servus salutem et gratiam in Christo Iesu atque coepti sacri ordinis nitidam perseveranter observare puritatem.

Sancto Spiritui gratias agimus, cuius instinctu circa canonicum ordinem vos, fratres, fervorem et studium habere cognoscimus.
Gelasius.

Gelasius servus servorum Dei omnibus in can[onico ordine secundum] Deum viventibus salutem et gratiam in Christo Iesu [atque coepti sacri ordinis] nitidam perseveranter observare puritatem.

Sancto [Spiritui gratias agimus], cuius instinctu circa canonicum ordinem vos, fr[atres], fervorem et studium habere cognoscimus.

Dann gehen beide verschiedene Wege. Letbert sucht vor allem aus dem Alten und Neuen Testament und durch allegorische Schriftauslegung die Würde des Ordens darzutun; erst gegen Ende geht er kurz auf seine Geschichte ein mit Worten, die unverkennbar an den Brieftext anklingen:

d'archéologie et de statistique de lá Jorôme IIJ, Valence 1868, S. 196 bis 214, habe ich nicht gesehen.

1) Chevalier S. 220 Anm. 5 weist auf die Landschaft Ferrain bei Lille und den dort gelegenen Ort Neuville-n-Ferain (cant. Tourcoing) hin.

2) Die Schrift haben Martènc und Durand herausgegeben, Thesaurus norus anecdotorum I, 1717, Sp. 329-332; danach Migne 157, $715-719$.

$\left.{ }^{3}\right)$ So die dem Druck zugrunde liegende Handschrift statt des urkundlich verbürgten 'Letbertus'. 
'Hic ordo noster, ut dixi, in Christo, in apostolis, in primitiva ecclesia primo exfloruit; sed frigescente caritate et instante persecutione, postmodum emarcuit. Quem Urbanus papa martyr deinde suis decretis coepit suscitare, beatus quoque Augustinus suis regulis ordinare, sanctus Hieronymus suis epistolis commendare ceterique sancti viri, quos longum est memorare', und er schließt mit den Worten von Matthäus 5, 16, die auch Gelasius und die verwandten Texte gegen Ende anführen. Nun hat Letbert bei diesen Stellen sich sicherlich an den Wortlaut eines solchen päpstlichen Schreibens angelehnt (wenn auch vielleicht unbewußt), und eben sein eigenes Stift hatte ja auch von Urban II. eine entsprechende, noch vorhandene Urkunde erhalten, die ihm natürlich bekannt gewesen ist. Aber daraus ist der Eingang seiner Schrift nicht entnommen, der auch in einen päpstlichen Schreiben jener Zeit einzig dastände, und man kann wohl kaum der Annahme ausweichen, daß Letbert ( $†$ 1110/11) hier Gelasius (1118/19) als Quelle gedient hat, indem man seine Anfangsworte mit dem Text der Lrkunde Urbans für St. Rufus (von 'Omnipotenti' an) zusammenschweißte. Aber ist dies wirklich im Auftrag des Papstes geschehen? Man kann daran erinnern, daß Gelasius in Oktober 1118 Italien verlassen und seine letzten Monate in Frankreich, vor allem in Südfrankreich zugebracht hat, wo er Mitte Dezember gerade in Avignon weilte ${ }^{1}$ ), und man könnte vermuten, der Wortlaut der Urkunde sei hier außerhalb der Kanzlei von den Augustinerchorherren von St. Rufus vorbereitet und von der Kanzlei unverändert beibehalten worden. Die Herkunft des Textes aus St. Rufus wird durch seine beiden Bestandteile sehr wahrscheinlich gemacht; aber für eine Papsturkunde dieser Art vermisse ich ein Gegenstück und kann mich nicht entschließen, einer wirklichen Urkunde des Gelasius die Beibehaltung einer solchen Grußformel zuzuschreiben. Handelt es sich aber um eine verfälschte Papsturkunde und ist der Name des Gelasius zu Unrecht an die Spitze gestellt, so ist wieder der Zweck

2) Vgl. Jaffé I². S. 778 ff. 
der Fälschung nicht recht ersichtlich, wenn man nicht zu der beliebten Annahme einer ,Stilübung" greifen will, die hier wenig befriedigen dürfte, und ich weiß auch nicht zu sagen, weshalb man die Fälschung gerade einem Papste mit so kurzem Pontifikat und dazu einem früheren Mönche wie Gelasius untergeschoben hat. Oder genügt zur Erklärung der Umstand, daß man von ihm zwei Urkunden in Angelegenheiten der Augustinerchorherren besa $B^{\text {? }}{ }^{1}$ ) So muß ich mit einem Fragezeichen schließen: möge ein Berufenerer die Antwort geben.

1) Vgl. oben S. 39. Die Annahme scheint mir kaum in Betracht zu kommen, daß eine Fälschung unter dem Namen von Gelasius dem Ersten (492-496) beabsichtigt worden ist, dem man, soweit ich sehe, erst lange nach dem 12. Jahrhundert die Einführung der Regel Augustins in den Lateran zugeschrieben hat; vgl. z. B. G. Pennottus, Generalis totius sacri ordinis clericorum canonicorum historia tripartita, Cöln 1645, S. 275 ff. und das Bullarium Canonicorum Regularium Congregationis Sanctissimi Salvatoris I, Rom 1733, S. 278. Bei Pennottus wird S. 276 eine Urkunde Eugens IV. (1431-47) für die Kanoniker des Laterans (vgl. L. Pastor, Geschichte der Päpste seit dem Ausgang des Mittelalters I 3.4, 1901, S. 341 Anm. 3) angeführt mit Angaben über die Geschichte der Regularkanoniker, die offenbar teilweise aus einer der Gelasiusurkunde und den verwandten Texten ähnlichen Quelle geschöpft sind. 